\title{
Weakly 2-Absorbing Ideals of So-Rings
}

\author{
${ }^{1 .}$ N. Ravi Babu ${ }^{2 .}$ Dr. T.V. Pradeep Kumar, ${ }^{3 .}$ Dr. P. V. SrinivasaRao \\ ${ }^{1,3}$ Associate Professor, Department of Basic Engineering, DVR \& Dr. HS MIC College of Technology, \\ Kanchikacherla- 521180, Krishna(District), Andhra Pradesh, India \\ 2. Assistant Professor, Department of Science \& Humanities, Acharya Nagarjuna University, Nagarjuna Nagar, \\ Guntur (District), Andhra Pradesh, India
}

*Corresponding Author: N. Ravi Babu, Associate Professor, Department of Basic Engineering, DVR \& Dr. HS MIC College of Technology, Kanchikacherla- 521180, Krishna(District), Andhra Pradesh, India

\begin{abstract}
A partial semiring is a structure possessing an infinitary partial addition and a binary multiplication, subject to a set of axioms. In this paper we introduce the notion of weakly 2-absorbing ideals in so-rings and studythe conditions under which a weakly 2-absorbing ideal is a 2-absorbing ideal. Also, we obtain various equivalent conditions on the weakly 2-absorbing ideals of Cartesian product of so-rings.
\end{abstract}

Keywords: Ideal, Prime ideal, 2-absorbing ideal, weakly 2-absorbing ideal, commutative so-ring.

\section{INTRODUCTION}

Partially defined infinitary operations occur in the contexts ranging from integration theory to programming language semantics. The general cardinal algebras studied by Tarski in 1949, Housdorff Topological commutative groups studied by Bourbaki in 1966, $\sum$ - structures studied by Higgs in 1980, sum-ordered partial monoids and sum-ordered partial semirings(so-rings) studied by Arbib, Manes and Benson [2], [4], and streenstrup [10] are some of the algebraic structures of the above type.

G.V.S. Acharyulu [8] and P.V.SrinivasaRao [6] developed the ideal theory for the sum-ordered partial semirings (so-rings). Continuing this study, in [9] \& [5] we introduced the notion of 2-absorbing ideals in so-rings and obtained their characteristics in a commutative so-ring. In this paper, we introduce the notion of weakly 2 -absorbingidealsin so-rings and obtain its characteristics in so-rings.

\section{Preliminaries}

In this section we collect some important definitions and results for our use in this paper.

2.1. Definition. [4] A partial Monoidis a pair ( $M, \sum$ ) where $\mathrm{M}$ is a non-empty set and $\sum$ is a partial addition defined on some, but not necessarily all families $\left(x_{i}: i \in I\right)$ in $\mathrm{M}$ subject to the following axioms:

(i) Unary Sum Axiom. If $\left(x_{i}: i \in I\right)$ is a one element family in $\mathrm{M}$ and $I=\{j\}$, then $\sum\left(x_{i}: i \in I\right)$ is defined and equals $x_{j}$.

(ii) Partion-Associativity Axiom. If $\left(x_{i}: i \in I\right)$ is a family in $\mathrm{M}$ and $\left(I_{j}: j \in J\right)$ is apartition of $\mathrm{I}$, then $\left(x_{i}: i \in I\right)$ is summable if and only if $\left(x_{i}: i \in I_{j}\right)$ is summablefor every $j \in J$ and $\left(\sum\left(x_{i}: i \in I_{j}\right): j \in J\right)$ is summable. We write $\sum\left(x_{i}: i \in I\right)=\Sigma\left(\sum\left(x_{i}: i \in I_{j}\right): j \in J\right)$.

2.2. Definition. [4] A Partial Semiringis a quadruple $\left(R, \sum, ., 1\right)$, where $\left(R, \sum\right)$ is a partial monoid, $(R, ., 1)$ is a monoid with multiplicative operation ' ' and unit 1 , and the additive and multiplicative 
structures obey the following distributive laws: If $\sum\left(x_{i}: i \in I\right)$ is defined in $\mathrm{R}$, then for all $\mathrm{y}$ in $R$, $\sum\left(y \cdot x_{i}: i \in I\right)$ and $\sum\left(x_{i} \cdot y: i \in I\right)$ are defined and $y \cdot \sum\left(x_{i}: i \in I\right)=\sum\left(y \cdot x_{i}: i \in I\right)$,

$\sum\left(x_{i}: i \in I\right) \cdot y=\sum\left(x_{i} \cdot y: i \in I\right)$.

2.3. Definition. [4] A partial semiring $\left(R, \sum, ., 1\right)$ is said to be commutativeif $x y=y x \forall x, y \in R$.

2.4. Definition. [4] The sum ordering $\leq$ on a partial monoid $(M, \Sigma)$ is the binary relation $\leq$ such that $x \leq y$ if and only if there exists a ' $h$ ' in M such that $y=x+h$ for $x, y \in M$.

2.5. Definition. [4] A sum-ordered partial semiring or so-ring, for short, is a partial semiring in which the sum ordering is a partial order.

2.6. Example. [4] Let $D$ be a set and let the set of all partial functions from D to D be denoted by $\operatorname{Pfn}(D, D)$. A family $\left(x_{i}: i \in I\right)$ is summable if and only if for $\mathrm{i}, \mathrm{j}$ in $\mathrm{I}$, and $i \neq j$, $\operatorname{dom}\left(x_{i}\right) \cap \operatorname{dom}\left(x_{j}\right)=\phi$. If $\left(x_{i}: i \in I\right)$ is summable then for any $\mathrm{d}$ in $\mathrm{D}$ $d\left(\sum_{i} x_{i}\right)=\left\{\begin{array}{c}d x_{i}, \quad \text { if } d \in d o m\left(x_{i}\right) \text { for some (unique) } i \in I ; \\ \text { undefined, otherwise. }\end{array}\right.$

And '.' defined as the usual functional composition, the ordering as the extension of functions and unit defined as the identity defined on D. Then $\left(\operatorname{Pfn}(D, D), \sum\right.$,. $)$ is a so-ring.

2.7. Example. [4] Let $\mathrm{D}$ be a set. A multi-function $x: D \rightarrow D$ maps each element in $\mathrm{D}$ to an arbitray subset of $\mathrm{D}$. Such multi-functions correspond bijectivelyto the relation $r \subseteq D \times D$, where $(d, e) \in r$ if and only if $e \in d x$. The set of all multi-functions from D to D, denoted by $\operatorname{Mfn}(D, D)$, together with $\sum$ defined such that $\mathrm{d}$ in $\mathrm{D}, d\left(\sum_{i} x_{i}\right)=\bigcup_{i}\left(d x_{i}\right)$, and ' ' defined as the usual relational composition. That is, for each $\mathrm{d}$ in $\mathrm{D}$ and for $\mathrm{x}, \mathrm{y}$ in $M f n(D, D), d(x \cdot y)=\bigcup(e y: e \in d x)$, and $d 1=\{d\}$. Then $\left(M f n(D, D), \sum,.\right)$ is a so-ring.

2.8. Definition. [8] Let $\mathrm{R}$ be a so-ring. A subset $\mathrm{N}$ of $\mathrm{R}$ is said to be an ideal of $\mathrm{R}$ if the following are satisified:

$\left(I_{1}\right)$. If $\left(x_{i}: i \in I\right)$ is a summable family in $\mathrm{R}$ and $x_{i} \in N \forall i \in I$ then $\sum\left(x_{i}: i \in I\right) \in N$,

$\left(I_{2}\right)$. If $x \leq y$ and $y \in N$ then $x \in N$,

$\left(I_{3}\right)$. If $x \in N$ and $r \in R$ then $x r, r x \in N$.

2.9. Definition. [7] A proper ideal $\mathrm{P}$ of a so-ring $\mathrm{R}$ is said to be weakly prime if for any $a, b$ of $\mathrm{R}$, $0 \# a b \in P$ imply $a \in P$ or $b \in P$.

2.10. Definition. [9]A proper ideal I of a so-ring $\mathrm{R}$ is said to be 2-absorbing if for any $a, b, c \in R$, $a b c \in I$ implies $a b \in I$ or $b c \in I$ or $a c \in I$.

2.11. Remark. [9] Every prime ideal of a so-ring $R$ is a 2-absorbing ideal ofR.

The following is an example of a so-ring $\mathrm{R}$ in which a 2-absorbing ideal need not be a prime ideal ofR.

2.12. Example [9]Consider the so-ring $R=\{0, u, v, x, y, 1\}$ with $\sum$ defined on $R$ by $\Sigma\left(x_{i}: i \in I\right)=\left\{\begin{array}{c}x_{j}, \quad \text { if } \quad x_{i}=0 \quad \forall i \neq j \text { for some } j, \\ \text { undefined, otherwise. }\end{array}\right.$

And '.' defined by the following table: 


\begin{tabular}{|c|c|c|c|c|c|c|}
\hline$\cdot$ & 0 & $\mathrm{u}$ & $\mathrm{v}$ & $\mathrm{x}$ & $\mathrm{y}$ & 1 \\
\hline 0 & 0 & 0 & 0 & 0 & 0 & 0 \\
\hline $\mathrm{u}$ & 0 & $\mathrm{u}$ & 0 & 0 & 0 & $\mathrm{u}$ \\
\hline $\mathrm{v}$ & 0 & 0 & $\mathrm{v}$ & 0 & 0 & $\mathrm{v}$ \\
\hline $\mathrm{x}$ & 0 & 0 & 0 & 0 & 0 & $\mathrm{x}$ \\
\hline $\mathrm{y}$ & 0 & 0 & 0 & 0 & 0 & $\mathrm{y}$ \\
\hline 1 & 0 & $\mathrm{u}$ & $\mathrm{v}$ & $\mathrm{x}$ & $\mathrm{y}$ & 1 \\
\hline
\end{tabular}

Then the ideal $I=\{0, u, x\}$ is a 2-absorbing ideal, but $\mathrm{I}$ is not a prime ideal. Since $v \cdot y=o \in I$, but $v \notin I$ and $y \notin I$.

Throughout this paper, $R$ denotes a commutative so-ring.

\section{WEAKLY 2-ABSORBING IDEALS}

We introduce the notion of weakly 2-absorbing ideals in so-rings as follows:

3.1. Definition. An ideal $\mathrm{I}$ of a so-ring $\mathrm{R}$ is said to be weakly 2-absorbing if for some $a, b, c \in R \&$ $0 \neq a b c \in I$, then $a b \in I$ or $b c \in I$ or $a c \in I$.

3.2. Remark. Every 2-absorbing ideal I of a so-ring R is a weakly 2-absorbing ideal of R.

The following is an example of a so-ring $\mathrm{R}$ in which a weakly 2-absorbing ideal is not a 2absorbing ideal of $\mathrm{R}$.

3.3. Example. Consider the so-ring $Z_{8}$. Take $R:=Z_{8} \times Z_{8}$. Then $\mathrm{R}$ is a commutative so-ring with respect to c0artesian product operations. Take $I:=\{(0,0),(0,4)\}$. Then it can be verified that $I$ is a weakly 2 -absorbing ideal of R. Since $(2,0)(2,0)(2,0)=(0,0) \in I$ and $(2,0)(2,0)=(4,0) \notin I$, I is not a 2-absorbing ideal of $\mathrm{R}$.

3.4. Theorem. Let $\mathrm{R}$ be a so-ring, $\mathrm{I}$ be an ideal of $\mathrm{R}$ and $a \in R$. Then the following statements are hold in R:

(i) Suppose $(0: a) \subseteq R a$, then the ideal $R a$ is 2-absorbing if and only if it is weakly 2-absorbing

(ii) Suppose $(0: a) \subseteq I a$, then the ideal $I a$ is 2-absorbing if and only if it is weakly 2-absorbing.

Proof.Let $a \in R$. (i) Assume that $(0: a) \subseteq R a$. Suppose $R a$ is a weakly 2 -absorbing ideal of R. Let $r, s, t \in R$ such that $r s t \in R a$. Suppose $r s t \neq 0$. Since $R a$ is weakly 2-absorbing, we have $r s \in R a$ or $s t \in R a$ or $r t \in R a$. Suppose $r s t=0$. Then $r(s+a) t=r s t+r a t=r a t=r t a \in R a$. Therefore $r(s+a) t \in R a$. If $r(s+a) t \neq 0$. Then $r(s+a) \in R a$ or $(s+a) t \in R a$ or $r t \in R a$ (Since $R a$ is weakly 2-absorbing). That implies $r s \in R a$ or $s t \in R a$ or $r t \in R a$. If $r(s+a) t=0$. Then $r s t+r a t=0$. That implies $r a t=0$. That implies $r t a=0$. That implies $r t \in(0: a) \subseteq R a$. That implies $r t \in R a$. Hence $R a$ is a 2-absorbing ideal of R. By Remark - 3.2., if $R a$ is a 2-absorbing ideal of $\mathrm{R}$ then $R a$ is a weakly 2-absorbing ideal of $R$.

(ii) Assume that $(0: a) \subseteq I a$. Suppose $I a$ is a weakly 2-absorbing ideal of R. Let $r, s, t \in R$ such that $r s t \in I a$. Suppose $r s t \neq 0$. Since $I a$ is weakly 2 -absorbing, we have $r s \in I a$ or $s t \in I a$ or $r t \in I a$. Suppose $r s t=0$. Then $0 r(s+a) t=r s t+r a t=r a t=r t a \in I a$. Therefore $r(s+a) t \in I a$. If $r(s+a) t \neq 0$. Then $r(s+a) \in I a$ or $(s+a) t \in I a$ or $r t \in I a$ (Since $I a$ is weakly 2-absorbing). That implies $r s \in I a$ or $s t \in I a$ or $r t \in I a$. If $r(s+a) t=0$. Then $r s t+r a t=0$. That implies $r a t=0$. That implies $r t a=0$. That implies $r t \in(0: a) \subseteq I a$. That implies $r t \in I a$. Hence $I a$ is a 2-absorbing ideal of R. By Remark - 3.2., if $I a$ is a 2-absorbing ideal of R then $I a$ is a weakly 2absorbing ideal of $R$. 
3.5. Theorem. IfI and $\mathrm{J}$ are weakly prime ideals of a so-ring $\mathrm{R}$, then $I \cap J$ is a weakly 2 -absorbing ideal of R.

Proof.Let $0 \neq a b c \in I \cap J$ for some $a, b, c \in R$. i.e., $0 \neq a b c \in I \& 0 \neq a b c \in J$. Since I,J are weakly prime ideals of $\mathrm{R}$, we have either $a \in I$ or $b c \in I \&$ either $a \in J$ or $b c \in J$. Suppose $b c=0$ then $b c \in I \cap J$, there is nothing to prove (Since $0 \in I \cap J$ ). So assume that $b c \neq 0$. Then either $a \in I$ or $0 \neq b c \in I \&$ either $a \in J$ or $0 \neq b c \in J$. That implies $a \in I$ or $b \in I$ or $c \in I \&$ $a \in J$ or $b \in J$ or $c \in J$ ( SinceI, Jare weakly prime ideals). That implies $a b \in I \cap J$ or $b c \in I \cap J$ or $a c \in I \cap J$. Hence $I \cap J$ is a weakly 2-absorbing ideal of R.

3.6. Definition. Let $\mathrm{I}$ be a weakly 2 -absorbing ideal of a so-ring $\mathrm{R}$ and $a, b, c \in R$. We say that $(a, b, c)$ is a triple-zeroif $a b c=0, a b \notin I, b c \notin I$ and $a c \notin I$.

3.7. Theorem. Let $\mathrm{I}$ be a weakly 2 -absorbing ideal of a so-ring $\mathrm{R}$ and suppose that $(a, b, c)$ is a triplezero of Ifor some $a, b, c \in R$. Then

(i) $a b I=b c I=a c I=\{0\}$

(ii) $a I^{2}=b I^{2}=c I^{2}=\{0\}$.

Proof.(i) In a contrary way suppose that $a b I \neq\{0\}$. i.e., $a b i \neq 0$ for some $i \in I$. That implies $a b(c+i) \neq 0$. Since $a b \notin I$ and $a b(c+i) \neq 0$, we have $a(c+i) \in I$ or $b(c+i) \in I$ (Since $\mathrm{I}$ is weakly 2-absorbing). i.e., $a c \in I$ or $b c \in I$, a contradiction to the fact that $(a, b, c)$ is a triple-zero. So our assumption is wrong. Hence $a b I=\{0\}$. Similarly we can prove that $b c I=\{0\}, a c I=\{0\}$.

(ii) In a contrary way suppose that $a I^{2} \neq\{0\}$. i.e., $a i_{1} i_{2} \neq 0$ for some $i_{1}, i_{2} \in I$. That implies $a\left(b+i_{1}\right)\left(c+i_{2}\right)=a i_{1} i_{2} \neq 0 \in I \quad$ (Since by (i), $a b I=b c I=a c I=\{0\}$ ). Since Iis a weakly 2absorbing ideal of R, either $a\left(b+i_{1}\right) \in I$ or $a\left(c+i_{2}\right) \in I$ or $\left(b+i_{1}\right)\left(c+i_{2}\right) \in I$. i.e., either $a b \in I$ or $b c \in I$ or $a c \in I$, a contradiction to the fact that $(a, b, c)$ is a triple-zero. So our assumption is wrong. Hence $a I^{2}=\{0\}$. Similarly we can prove that $b I^{2}=c I^{2}=\{0\}$.

\section{WEAKLY 2-ABSORBING IDEALS IN CARTESIAN PRODUCTS}

4.1. Theorem. Let $R_{1}$ and $R_{2}$ be so-rings and $\mathrm{I}$ be a proper ideal of $R_{1}$. Then the following conditions are equivalent:

(i) I is a weakly 2-absorbing ideal of $R=R_{1} \times R_{2}$,

(ii) $I \times R_{2}$ is a weakly 2-absorbing ideal of $R=R_{1} \times R_{2}$.

Proof.(i) $\Rightarrow$ (ii): Suppose I is a weakly 2-absorbing ideal of R. Let $\left(a_{1}, a_{2}\right),\left(b_{1}, b_{2}\right),\left(c_{1}, c_{2}\right) \in R=R_{1} \times R_{2} \quad$ such that $0 \neq\left(a_{1}, a_{2}\right)\left(b_{1}, b_{2}\right)\left(c_{1}, c_{2}\right) \in I \times R_{2}$. Then $0 \neq\left(a_{1} b_{1} c_{1}, a_{2} b_{2} c_{2}\right) \in I \times R_{2}$. Therefore $0 \neq a_{1} b_{1} c_{1} \in I$. Since I is a weakly 2 -absorbing ideal of $\mathrm{R}$, $a_{1} b_{1} \in I$ or $b_{1} c_{1} \in I$ or $a_{1} c_{1} \in I$. If $a_{1} b_{1} \in I$ then $\left(a_{1}, a_{2}\right)\left(b_{1}, b_{2}\right) \in I \times R_{2}$. If $b_{1} c_{1} \in I$ then $\left(b_{1}, b_{2}\right)\left(c_{1}, c_{2}\right) \in I \times R_{2}$. If $a_{1} c_{1} \in I$ then $\left(a_{1}, a_{2}\right)\left(c_{1}, c_{2}\right) \in I \times R_{2}$. Hence $I \times R_{2}$ is a weakly 2absorbing ideal of $R=R_{1} \times R_{2}$.

(ii) $\Rightarrow$ (i): Suppose $I \times R_{2}$ is a weakly 2-absorbing ideal of $R=R_{1} \times R_{2}$. Let $0 \neq a b c \in I$ for some $a, b, c \in R$. Then for each $0 \neq r \in R_{2}$, we have $0 \neq(a, 1)(b, 1)(c, r) \in I \times R_{2}$. Since $I \times R_{2}$ is a weakly 2-absorbing ideal of $R=R_{1} \times R_{2}, \quad(a, 1)(b, 1) \in I \times R_{2} \quad$ or $\quad(b, 1)(c, r) \in I \times R_{2}$ or $(a, 1)(c, r) \in I \times R_{2}$. That implies $a b \in I$ or $b c \in I$ or $a c \in I$. Hence I is a weakly 2-absorbing ideal of R. 
4.2. Theorem. Let $R=R_{1} \times R_{2}$ where $R_{1}$ and $R_{2}$ are so-rings. Let $\mathrm{I}$ be a proper ideal of $R_{1}$ and $\mathrm{J}$ be a proper ideal of $R_{2}$. Then the following statements are equivalent:

(i) $I \times J$ is a weakly 2 -absorbing ideal of R,

(ii) ( $J=R_{2}$ and I is a weakly 2-absorbing ideal of $R_{1}$ ) or ( $\mathrm{J}$ is a prime ideal of $R_{2}$ and $\mathrm{I}$ is a prime ideal of $R_{1}$ ).

Proof.(i) $\Rightarrow$ (ii): Suppose $I \times J$ is a weakly 2-absorbing ideal of R. If $J=R_{2}$ then $I \times R_{2}$ is a weakly 2 -absorbing ideal of $\mathrm{R}$. Then by theorem 4.2., I is a weakly 2 -absorbing ideal of $R_{1}$. Suppose $J \neq R_{2}$. We have to prove that $\mathrm{J}$ is a prime ideal of $R_{2}$ and $\mathrm{I}$ is a prime ideal of $R_{1}$. Let $a, b \in R_{2}$ such that $a b \in J$, and let $0 \neq i \in I$. Then $0 \neq(i, 1)(1, a)(1, b)=(i, a b) \in I \times J$. Now $(1, a)(1, b)=(1, a b) \notin I \times J$ (Since $1 \notin I)$. Since $I \times J$ is a weakly 2 -absorbing ideal of R, either $(i, 1)(1, a)=(i, a) \in I \times J$ or $(i, 1)(1, b)=(i, b) \in I \times J$. That implies either $a \in J$ or $b \in J$. Hence $\mathrm{J}$ is a prime ideal of $R_{2}$. Similarly let $c, d \in R_{1}$ such that $c d \in I$, and let $0 \neq j \in J$. Then $0 \neq(c, 1)(d, 1)(1, j)=(c d, j) \in I \times J$. Now $(c, 1)(d, 1)=(c d, 1) \notin I \times J($ Since $1 \notin J)$. Since $I \times J$ is a weakly 2-absorbing ideal of R, either $(c, 1)(1, j)=(c, j) \in I \times J$ or $(d, 1)(1, j)=(d, j) \in I \times J$. That implies either $c \in I$ or $d \in I$. Hence I is a prime ideal of $R_{1}$.

(ii) $\Rightarrow$ (i): Suppose $J=R_{2}$ and $\mathrm{I}$ is a weakly 2-absorbing ideal of $R_{1}$ or $\mathrm{J}$ is a prime ideal of $R_{2}$ and I is a prime ideal of $R_{1}$. We have to prove that $I \times J$ is a weakly 2-absorbing ideal of R. Suppose $J=R_{2} \& \mathrm{I}$ is a weakly 2 -absorbing ideal of $R_{1}$, by theorem 4.2., $I \times R_{2}$ is a weakly 2 -absorbing ideal of R. i.e., $I \times J$ is a weakly 2 -absorbing ideal of R. SupposeJ is a prime ideal of $R_{2} \& \mathrm{I}$ is a prime ideal of $R_{1}$. Let $0 \neq\left(a_{1}, b_{1}\right)\left(a_{2}, b_{2}\right)\left(a_{3}, b_{3}\right) \in I \times J$ for some $a_{1}, a_{2}, a_{3} \in R_{1}$ and $b_{1}, b_{2}, b_{3} \in R_{2}$. Then $a_{1} \in I$ or $a_{2} \in I$ or $a_{3} \in I$ and $b_{1} \in J$ or $b_{2} \in J$ or $b_{3} \in J$. Thus $\left(a_{1}, b_{1}\right)\left(a_{2}, b_{2}\right) \in I \times J$ or $\left(a_{2}, b_{2}\right)\left(a_{3}, b_{3}\right) \in I \times J$ or $\left(a_{1}, b_{1}\right)\left(a_{3}, b_{3}\right) \in I \times J$. Hence $I \times J$ is a weakly 2-absorbing ideal of R.

4.3. Theorem. Let $R_{1}, R_{2}$ be a so-rings such that $R_{2}$ has no nonzero divisors. Let I be a proper ideal of $R_{1}$ and $\mathrm{J}$ be an ideal of $R_{2}$. Then the following statements are equivalent:

(i) $I \times J$ is a weakly 2-absorbing ideal of $R=R_{1} \times R_{2}$,

(ii) I is a weakly prime ideal of $R_{1}$ and $J=\{0\}$ is a prime ideal of $R_{2}$.

Proof.(i) $\Rightarrow$ (ii): Suppose $I \times J$ is a weakly 2 -absorbing ideal of R. Suppose $J=\{0\}$. We have to prove that $J=\{0\}$ is a prime ideal of $R_{2}$. Let $a b \in J=\{0\}$ for some $a, b \in R_{2}$. Let $0 \neq i \in I$, we have $0 \neq(i, 1)(1, a)(1, b)=(i, a b) \in I \times J$. Also we have $(1, a)(1, b)=(1, a b) \notin I \times J \quad($ Since $1 \notin I)$. Since $I \times J$ is a weakly 2-absorbing ideal of $\mathrm{R}$, either $(i, 1)(1, a)=(i, a) \in I \times J$ or $(i, 1)(1, b)=(i, b) \in I \times J$. That implies either $a \in J$ or $b \in J$. Hence $J=\{0\}$ is a prime ideal of $R_{2}$ . Now we have to prove that $\mathrm{I}$ is a weakly prime ideal of $R_{1}$ that is not a prime ideal. Suppose $0 \neq a b \in I \quad$ for $\quad$ some $a, b \in R_{1}$. We have $0 \neq(a, 1)(b, 1)(1,0)=(a b, 0) \in I \times\{0\}$. Since $(a, 1)(b, 1)=(a b, 1) \notin I \times\{0\} \& I \times\{0\} \quad$ is a weakly 2 -absorbing ideal of $\mathrm{R}$, either $(a, 1)(1,0)=(a, 0) \in I \times\{0\}$ or $(b, 1)(1,0)=(b, 0) \in I \times\{0\}$. That implies either $a \in I$ or $b \in I$. Hence I is a weakly prime ideal of $R_{1}$.

(ii) $\Rightarrow$ (i): Suppose I is a weakly prime ideal of $R_{1}$ that is not a prime ideal $\& J=\{0\}$ is a prime ideal of $R_{2}$. We have to prove that $I \times\{0\}$ is a weakly 2-absorbing ideal of $\mathrm{R}$. Let $0 \neq(a, b)(c, d)(e, f)=(a c e, b d f) \in I \times\{0\}$. Since I is a weakly prime ideal of $R_{1}$, we may assume 
that $a \in I$. Since $R_{2}$ has no nonzero divisors, we may assume that $d=0$. Therefore $(a, b)(c, d)=(a, b)(c, 0)=(a c, 0) \in I \times\{0\}$. Hence $I \times\{0\}$ is a weakly 2 -absorbing ideal of R.

4.4. Theorem. Let $R=R_{1} \times R_{2} \times R_{3}$ where $R_{1}, R_{2}, R_{3}$ are so-rings. Let $I_{1}$ be a proper ideal of $R_{1}, I_{2}$ be an ideal of $R_{2}$, and $I_{3}$ be an ideal of $R_{3}$ such that $I=I_{1} \times I_{2} \times I_{3} \neq\{(0,0,0)\}$. Then the following statements are equivalent:

(i) $I=I_{1} \times I_{2} \times I_{3}$ is a weakly 2-absorbing ideal of R,

(ii) $I=I_{1} \times R_{2} \times R_{3}$ and $I_{1}$ is a weakly 2-absorbing ideal of $R_{1}$ or $I=I_{1} \times I_{2} \times R_{3}$ such that $I_{1}$ is a prime ideal of $R_{1}$ and $I_{2}$ is a prime ideal of $R_{2}$ or $I=I_{1} \times R_{2} \times I_{3}$ such that $I_{1}$ is a prime ideal of $R_{1}$ and $I_{3}$ is a prime ideal of $R_{3}$.

Proof. (i) $\Rightarrow$ (ii): Suppose $I=I_{1} \times I_{2} \times I_{3}$ is a weakly 2-absorbing ideal of R. Since I is a weakly 2absorbing ideal of $\mathrm{R}, I_{1}$ is a weakly 2-absorbing ideal of $R_{1}$. If $I_{2}=R_{2}$ and $I_{3}=R_{3}$, then $I=I_{1} \times R_{2} \times R_{3}$. Suppose $I_{2} \neq R_{2} \& I_{3}=R_{3}$. i.e., $I=I_{1} \times I_{2} \times R_{3}$. Now we have to prove that $I_{1}$ is a prime ideal of $R_{1}$ and $I_{2}$ is a prime ideal of $R_{2}$. Let $a, b \in R_{1}$ such that $a b \in I_{1}$ and $c, d \in R_{2}$ such that $c d \in I_{2}$. Then $0 \neq(a, 1,1)(1, c d, 1)(b, 1,1)=(a b, c d, 1) \in I$. Now $(a, 1,1)(b, 1,1)=(a b, 1,1) \notin I$ (Since $I=I_{1} \times I_{2} \times R_{3}$ and $1 \notin I_{2}$ ). SinceI is a weakly 2-absorbing ideal of $\mathrm{R}$, we have either $(a, 1,1)(1, c d, 1)=(a, c d, 1) \in I$ or $(1, c d, 1)(b, 1,1)=(b, c d, 1) \in I$. That implies either $a \in I_{1}$ or $b \in I_{1}$. Hence $I_{1}$ is a prime ideal of $R_{1}$. Similarly $0 \neq(a b, 1,1)(1, c, 1)(1, d, 1)=(a b, c d, 1) \in I$. Now $(1, c, 1)(1, d, 1)=(1, c d, 1) \notin I$ (Since $\left.I=I_{1} \times I_{2} \times R_{3} \& 1 \notin I_{1}\right)$. SinceI is a weakly 2 -absorbing ideal of $\mathrm{R}$, we have either $(a b, 1,1)(1, c, 1)=(a b, c, 1) \in I$ or $(a b, 1,1)(1, d, 1)=(a b, d, 1) \in I$. That implies either $c \in I_{2}$ or $d \in I_{2}$. Hence $I_{2}$ is a prime ideal of $R_{2}$. Finally assume that $I_{2}=R_{2}$ and $I_{3} \neq R_{3}$ (i.e., $I=I_{1} \times R_{2} \times I_{3}$ ). By applying the above arguement, we conclude that $I_{1}$ is a prime ideal of $R_{1}$ and $I_{3}$ is a prime ideal of $R_{3}$.

(ii) $\Rightarrow$ (i): Suppose $\mathrm{I}$ is one of the given three forms. Then by theorem 4.2., $I=I_{1} \times I_{2} \times I_{3}$ is a weakly 2-absorbing ideal of R.

\section{CONCLUSiON}

In this paper we introduced the notion of weakly 2-absorbing ideals in so-rings and provided a counter example that proves the class of weakly 2-absorbing ideals is strictly wider than the class of all 2absorbing ideals. Also we obtained the conditions under which a weakly 2-absorbing ideal is a 2absorbing ideal. We considered this notation of weakly 2-absorbing ideals in the Cartesian product of so-rings and obtained various equivalent conditions on the weakly 2 -absorbing ideals of Cartesian product of so-rings.

\section{REFERENCES}

[1] Prathibha Kumar, Manish Kant Dubey and PoonamSarohe.,Some results on 2-absorbing ideals in Commutative Semirings, Journal of Mathematics and Applications.38, 77-84 (2015).

[2] Arbib, M.A., Manes, E.G.,Partially Additive Categories and Flow-diagram Semantics, Journal of Algebra.62, 203-227(1980).

[3] Chaudhari J.N.,2-absorbing Ideals in Semirings, International Journal of Algera. 6(6), 265-270 (2012).

[4] Manes, E.G., and Benson, D.B.,The Inverse Semigroup of a Sum-Ordered Partial Semirings, Semigroup Forum. 31, 129-152 (1985).

[5] Ravi Babu, N., Pradeep Kumar, T.V., and SrinivasaRao, P.V., 2-absorbing ideals in so-rings (communicated to International Journal of Pure and Applied Mathematics).

[6] SrinivasaRao, P.V., Ideals Of Sum-ordered Semirings, International Journal of Computational Cognition (IJCC). 7(2), 59-64 (2009). 
[7] Srinivasa Reddy, M., AmarendraBabu, V., SrinivasaRao, P.V., 2-absorbing Subsemimodules of Partial Semimodules, Gen.Math.Notes.23(2), 43-50(2014).

[8] Acharyulu, G.V.S., A Study of Sum-Ordered Partial Semirings, Doctoral thesis, Andhra University.(1992).

[9] SrinivasaRao, P.V., Ideal Theory of Sum-ordered Partial Semirings, Doctoral thesis, AcharyaNagarjuna University.(2011).

[10] Streenstrup, M.E., Sum-ordered Partial Semirings, Doctoral thesis, Graduate school of the University of Massachusetts. (1985) (Department of computer and Information Science).

\section{AUTHOR's BIOGRAPHY}

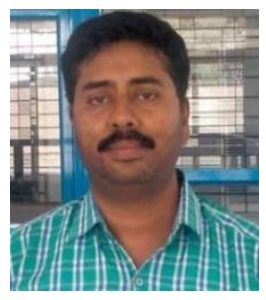

He is working as an Assistant Professor in the Department of Basic Engineering, DVR \&Dr. HS MIC College of Technology. He is pursuing Ph. D in Acharya Nagarjuna University under the guidance Dr. T.V. Pradeep Kumar. Presently he is working on So-rings.

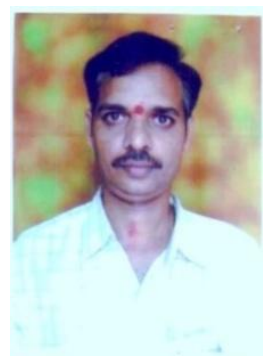

He is working as an Assistant Professor in the Department of Science \& Humanities, ANU Engineering College, Acharya Nagarjuna University. He received the Ph. D degree on the work "Contributuion to Near Ring Theory-III" under the guidance Prof. Bhavanari Satyanarayana from Acharya Nagarjuna University in 2008. He has presented papers in various seminars and published more than 25 research papers in popular International Journals to his credit. His area of interests are Algebra, Graph Theory, Topology, Analysis, Fuzzy Sets. Presently he is working on Algebra, Graph Theory, Topology, Fuzzy Sets.

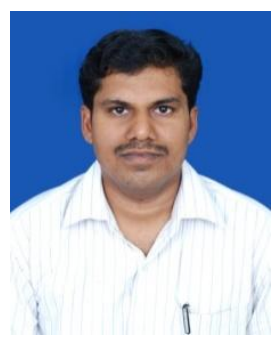

He is working as an Associate Professor in the Department of Basic Engineering, DVR \&Dr. HS MIC College of Technology. He received the Ph. D degree on the work "Ideal Theory of Sum-ordered Partial Semirings" under the guidance Dr. N. PrabhakaraRao from AcharyaNagarjuna University in 2012. He has presented papers in various seminars and published more than 21 research papers in popular International Journals to his credit. His area of interests areSemirings, $\Gamma$ semirings and Ordered algebras. Presently he is working on Partial Semirings, Partial $\Gamma$-semirings\& Ternary Semirings.

Citation: N. H. Shah et al., " Weakly 2-Absorbing Ideals of So-Rings ", International Journal of Scientific and Innovative Mathematical Research, vol. 5, no. 7, p. 29-35, 2017., http://dx.doi.org/10.20431/ 23473142.0507003

Copyright: () 2017 Authors. This is an open-access article distributed under the terms of the Creative Commons Attribution License, which permits unrestricted use, distribution, and reproduction in any medium, provided the original author and source are credited. 